\title{
Photon position measure
}

\author{
Margaret Hawton* \\ Department of Physics, Lakehead University, Thunder Bay, ON, Canada, P7B 5E1
}

\begin{abstract}
The positive operator valued measure (POVM) for a photon counting array detector is derived and found to equal photon flux density integrated over pixel area and measurement time. Since photon flux density equals number density multiplied by the speed of light, this justifies theoretically the observation that a photon counting array provides a coarse grained measurement of photon position. The POVM obtained here can be written as a set of projectors onto a basis of localized states, consistent with the description of photon position in a recent quantum imaging proposal [M. Tsang, Phys. Rev. Lett. 102, 253601 (2009)]. The wave function that describes a photon counting experiment is the projection of the photon state vector onto this localized basis. Collapse is to the electromagnetic vacuum and not to a localized state, thus violating the text book rules of quantum mechanics but compatible with the theory of generalized observables and the nonlocalizability of an incoming photon.
\end{abstract}

\section{INTRODUCTION}

Although position of a massive particle is an observable in nonrelativistic quantum mechanics, photon position is a controversial concept. It has been argued that there is no photon number density, only energy density [1]. Solutions to the photon wave equation are electric and magnetic fields, but the relationship between these fields and the normalizable Laudau-Peierls (LP) photon number amplitude is nonlocal [2, 3]. Localization of a converging (or diverging) photon pulse cannot be exact since, according to the Paley-Weiner theorem, it must have subexponential tails [4].

The most appropriate real space description of the photon is a subject of ongoing debate. Since photons are detected through interaction with matter and atomic dipoles are sensitive to electric field, the positive frequency part of this field is often called the photon wave function [5]. A LP-like wave function has been defined in the context of the inverse problem, spontaneous emission of a photon by an excited atom [6]. A measurement of photon position should be described by projection onto a basis of localized photon states [7], but this probability amplitude is not a positive frequency electromagnetic field.

In spite of this theoretical controversy, a single photon is counted in only one pixel of an array detector and there is considerable interest in the measurement of photon position in quantum optics. Position entanglement has been demonstrated experimentally to be a way to achieve high dimensional entangled states for information processing [8]. Recently Tsang defined a photon POVM and applied it to a new quantum imaging method [9]. The use of a POVM to describe photon position was introduced first by Kraus [10].

Use of a POVM bypasses the theoretical difficulties because a POVM is just an operator partition of the iden-

*Electronic address: margaret.hawton@lakeheadu.ca tity that does not require a self-adjoint position operator. Here a photon position POVM is obtained by lifting it to a larger Hilbert space that includes the atoms of a photodetector. We will start by considering absorption of photons by a two dimensional (2D) photon counting array detector. This experiment is described by a projection valued measure (PVM) that follows the usual rules for an observable in quantum mechanics. Projection operators onto each pixel will be defined such that their expectation values equal the probability to create an electron-hole pair. We will then examine how this photon counting experiment gives information about photon position.

\section{THEORY}

In the theory of generalized observables a POVM is an operator partition of the identity that describes the possible outcomes of a measurement. According to Neumark's dilation theorem a POVM on the Hilbert space $\mathcal{P}$ can always be lifted to a PVM on a bigger Hilbert space, $\mathcal{H}=\mathcal{P} \otimes \mathcal{A}$, where $\mathcal{A}$ is an ancillary subspace. The operators associated to a measurement on $\mathcal{H}, \widehat{F}_{n}$, are then a set of self-adjoint projection operators satisfying the usual postulates of quantum mechanics. The POVM on $\mathcal{P}$ is the partial trace on the ancillary Hilbert subspace, $\mathcal{A}$, that is

$$
\widehat{P}_{n}=\operatorname{Tr}_{\mathcal{A}}\left(\widehat{\rho}_{\mathcal{A}} \widehat{F}_{n}\right) .
$$

This formalism will be applied to a photon counting measurement: In Subsection A the localized states needed to define photon density will be discussed. In B, time dependent photodetection operators will be derived, and in $\mathrm{C}$ the special case of a thick detector that counts photons will be considered. Limitation of the model are discussed in $\mathrm{D}$, and in $\mathrm{E}$ and $\mathrm{F}$ the counting operators will be applied to one and two photon states and collapse. Normal incidence and the paraxial limit will be assumed so that cross talk between pixels can be neglected. 


\section{A. Localized states}

A state with definite position is an equally weighted sum over all momenta. States of this form were used by Tsang to describe photon position 99]. Here periodic boundary conditions over the volume $V$ will be used so that the momenta, $\mathbf{k}$, are discrete. Since all momenta are included, the two transverse polarizations should be defined for all wave vectors. In $\mathbf{k}$-space spherical polar coordinates the definite helicity unit vectors $\mathbf{e}_{\mathbf{k}, \sigma}^{(0)}=(\widehat{\theta}+i \sigma \widehat{\phi}) / \sqrt{2}$ can be defined for $\sigma= \pm 1$. In the paraxial approximation the basis states are then circularly polarized $(\mathrm{CP})$. The most general transverse basis vectors, $\mathbf{e}_{\mathbf{k}, \sigma}^{(\chi)}=\exp (-i \sigma \chi) \mathbf{e}_{\mathbf{k}, \sigma}^{(0)}(\theta, \phi)$, are rotated about $\mathbf{k}$ through the Euler angle $\chi(\theta, \phi)$ 11]. For example, if $\chi=-\phi$ the unit vectors are rotated back to the $x$ and $y$-axes and describe linearly polarized (LP) photons in the paraxial limit.

If the operator $\widehat{a}_{\mathbf{k}, \sigma}$ annihilates a photon with wave vector $\mathbf{k}$ and helicity $\sigma$, the operator

$$
\widehat{a}_{\sigma}(\mathbf{r}, t)=\frac{1}{\sqrt{V}} \sum_{\mathbf{k}} \widehat{a}_{\mathbf{k}, \sigma} \exp (i \mathbf{k} \cdot \mathbf{r}-i k c t)
$$

annihilates a photon with helicity $\sigma$ at position $\mathbf{r}$ and time $t$. The one-photon localized states are

$$
|\mathbf{r}, t, \sigma\rangle=\widehat{a}_{\sigma}^{\dagger}(\mathbf{r}, t)|\emptyset\rangle
$$

where $\widehat{a}_{\sigma}^{\dagger}$ is the adjoint of $\widehat{a}_{\sigma}$ and $|\emptyset\rangle$ is the electromagnetic vacuum state. This is an orthonormal one-photon basis at any fixed time, $t$. The number density operator is

$$
\widehat{n}(\mathbf{r}, t)=\sum_{\sigma} \widehat{a}_{\sigma}^{\dagger}(\mathbf{r}, t) \widehat{a}_{\sigma}(\mathbf{r}, t)
$$

\section{B. Photodetection}

It is assumed for concreteness that the photodetector is made up of a 2D array of semiconductor pixels with the $z$-axis chosen parallel to its inward normal. The dipole moment of the individuals atoms is taken to be isotropic with components all equal to $\mu$. The atoms are assumed to lie on a lattice so the atom positions are also discrete. The area, $A$, and the time, $T$, occupied by the electromagnetic pulse to be detected are taken to be finite so that $V=c T A$. To obtain time dependent operators that describe photon annihilation the Schrödinger picture (SP) operators will be transformed to the interaction picture (IP) and then to the Heisenberg picture (HP).

Glauber defined "an ideal photon detector as a system of negligible size (e.g., of atomic or subatomic dimensions) which has a frequency-independent photoabsorption probability" [12]. Detection of a photon with polarization in the $p$-direction by a Glauber photodetector at $\mathbf{r}$ will be described by the SP projection operator $\left|e_{\mathbf{r}, p}\right\rangle\left\langle e_{\mathbf{r}, p}\right|$ in the localized Wannier basis satisfying $\left\langle e_{\mathbf{r}, p} \mid e_{\mathbf{r}^{\prime}, p^{\prime}}\right\rangle=\delta_{\mathbf{r}, \mathbf{r}^{\prime}} \delta_{p, p^{\prime}}$ [13]. The factor $\delta_{p, p^{\prime}}$ describes absorption of a photon with definite polarization, as would be the case for a spherically symmetric hydrogen-like atom making a transition from a $n s$ to a $(n+1) p_{x}$, $(n+1) p_{y}$ or $(n+1) p_{z}$ state. For normal incidence in the paraxial approximation we can choose $\widehat{\mathbf{z}}$ parallel to the direction of propagation of the beam and $p=\sigma$ for any wave vector that is actually present in the pulse. In this case we will only need $\widehat{a}_{\sigma}(\mathbf{r}, t)$ defined by (2), although more generally $\widehat{a}_{\mathbf{k}, p}=\sum_{\sigma} \widehat{a}_{\mathbf{k}, \sigma}\left(\mathbf{e}_{\mathbf{k}, \sigma} \cdot \mathbf{e}_{p}^{*}\right)$ where $\mathbf{e}_{p}$ is a mutually orthogonal triad of unit vectors.

The SP counting operators are

$$
\widehat{F}_{0}^{S P}=\widehat{1}_{\mathcal{A}} \otimes \widehat{1}_{\mathcal{P}}=\widehat{1}
$$

for zero photons,

$$
\widehat{F}_{1, n}^{S P}=\sum_{p, \mathbf{r} \in D_{n}}\left|e_{\mathbf{r}, p}\right\rangle\left\langle e_{\mathbf{r}, p}\right| \otimes \widehat{1}_{\mathcal{P}}
$$

for one photon in pixel $n$,

$$
\widehat{F}_{2, n, n^{\prime}}^{S P}=\sum_{p, \mathbf{r} \in D_{n} ; p^{\prime}, \mathbf{r}^{\prime} \in D_{n^{\prime}}}\left|e_{\mathbf{r}^{\prime}, p^{\prime}}\right\rangle\left|e_{\mathbf{r}, p}\right\rangle\left\langle e_{\mathbf{r}, p}\right|\left\langle e_{\mathbf{r}^{\prime}, p^{\prime}}\right| \otimes \widehat{1}_{\mathcal{P}}
$$

for two photons, one in pixel $n$ and one in pixel $n^{\prime} \neq n$, and so on. The sums are over polarization and all atoms in the $n^{t h}$ pixel of the photodetector and $\left\{\mathbf{r} \in D_{n}\right\}$ are the positions of the atoms at which an elecron-hole (e-h) pair can be created. The unit operator, $\widehat{1}_{\mathcal{P}}$, acting in the photon Hilbert subspace $\mathcal{P}$, acknowledges the fact that no information is obtained directly from the photons.

These operators will be transformed to the IP and then to the HP. The SP Hamiltonian is

$$
\widehat{H}^{S P}=\widehat{H}_{0}+\widehat{H}_{I}^{S P}+\widehat{H}_{I}^{S P \dagger}
$$

with zero order term $\widehat{H}_{0}$ and photon-atom interaction described by

$$
\widehat{H}_{I}^{S P}=\mu \sum_{p, \mathbf{r} \in V} \widehat{E}_{\sigma}^{(+) S P}(\mathbf{r})\left|e_{\mathbf{r}, p}\right\rangle\langle g| .
$$

In (9),,$\left|e_{\mathbf{r}, p}\right\rangle\langle g|$ creates an e-h pair at $\mathbf{r}$ with atomic dipole moment in the $p$-direction. The SP positive frequency electric field operator is

$$
\widehat{E}_{p}^{(+) S P}(\mathbf{r})=i \sum_{\mathbf{k}} E_{k} \exp (i \mathbf{k} \cdot \mathbf{r}) \widehat{a}_{\mathbf{k}, p}
$$

where, with $k \equiv|\mathbf{k}|$,

$$
E_{k}=\sqrt{\frac{\hbar k c}{2 \epsilon_{0} V}}
$$

In the IP and within the rotating wave approximation $\widehat{H}_{0}$ is unchanged but the interaction Hamiltonian 
becomes time dependent. In a semiconductor the atoms interact, so the Wannier states, $\left|e_{\mathbf{r}, p}\right\rangle$, do not have definite energy. The eigenvectors of the atomic part of $\widehat{H}_{0}$ are the Bloch basis states, $\left|\psi_{\mathbf{q}, p}\right\rangle$, and its eigenvalues form a band of energies, $\epsilon_{q}$, where $\epsilon_{q}$ equals to the gap energy plus the energy of the electron hole pair. The $N$-atom Wannier basis is related to the Bloch basis according to [13]

$$
\left|e_{\mathbf{r}, p}\right\rangle=\frac{1}{\sqrt{N}} \sum_{\mathbf{q}} \exp (i \mathbf{q} \cdot \mathbf{r})\left|\psi_{\mathbf{q}, p}\right\rangle
$$

For a cubic lattice using periodic boundary conditions the wave vector $\mathbf{q}$ has components $q_{i}=2 \pi n_{i} / a N_{i}$ for $n_{i}=1$ to $N_{i}$ where $a$ is the interatomic distance and $N_{x} N_{y} N_{z}=N$ is the number of atoms in the pixel. For the photon, the expansion (10) gives states with definite energy, $k c$. Thus in (9) $\widehat{a}_{\mathbf{k}, p}\left|\psi_{\mathbf{q}, p}\right\rangle\langle g|$ acquires a time dependence $\exp \left[i\left(\epsilon_{q}-k c\right) t\right]$ in the IP [14], equivalent to the unitary transformation $\widehat{O}^{I P}=\widehat{U}_{0}^{\dagger} \widehat{O}^{S P} \widehat{U}_{0}$ with

$$
\widehat{U}_{0}(t)=\exp \left(-i \widehat{H}_{0} t / \hbar\right) .
$$

We can transform from the IP to the HP by solving $i \hbar d \widehat{U} / d t=\widehat{H}_{I} \widehat{U}$ iteratively to give the Dyson series [15]

$$
\widehat{U}(t)=\widehat{1}+\widehat{U}^{(1)}(t)+\widehat{U}^{(2)}(t)+\ldots
$$

where

$$
\begin{gathered}
\widehat{U}^{(1)}(t)=-\frac{i}{\hbar} \int_{t_{0}}^{t} d t^{\prime} \widehat{H}_{I}^{I P}\left(t^{\prime}\right), \\
\widehat{U}^{(2)}(t)=-\frac{1}{\hbar^{2}} \int_{t_{0}}^{t} d t^{\prime} \widehat{H}_{I}^{I P}\left(t^{\prime}\right) \int_{t_{0}}^{t^{\prime}} d t^{\prime \prime} \widehat{H}_{I}^{I P}\left(t^{\prime \prime}\right),
\end{gathered}
$$

and it is assumed that the detector is in its ground state at time $t_{0}$. The Hermitian conjugate has been omitted since photon emission by the semiconductor will be neglected.

All photons incident on an ideal photon counting detector must be absorbed, by definition of such a detector. Thus an $J$-photon counting operator must reduce a $J$-photon state to the vacuum state, $|\emptyset\rangle$. This is the case for terms of the form

$$
\widehat{F}_{J}^{H P}=\widehat{U}^{(J) \dagger}(t) \widehat{F}_{J}^{I P} \widehat{U}^{(J) \dagger}(t) .
$$

In the HP the zero photon operator remains $\hat{1}$, and the one-photon operator is

$$
\begin{aligned}
\widehat{F}_{1, n}^{H P}\left(t_{0}\right) & =\widehat{U}^{(1) \dagger}(t) \widehat{F}_{1, n}^{I P}(t) \widehat{U}^{(1)}(t) \\
& =\frac{|\mu|^{2}}{\hbar^{2}} \sum_{p ; \mathbf{r}, \mathbf{r}^{\prime}, \mathbf{r}^{\prime \prime} \in D_{n}} \int_{t_{0}}^{t_{0}+\Delta t} d t^{\prime} \int_{t_{0}}^{t_{0}+\Delta t} d t^{\prime \prime} \\
& \times \widehat{U}_{0}^{\dagger}\left(t^{\prime}\right)|g\rangle\left\langle e_{\mathbf{r}^{\prime}, p}\left|\widehat{E}_{p}^{(-) S P}\left(\mathbf{r}^{\prime}\right) \widehat{U}_{0}\left(t^{\prime}\right)\right| e_{\mathbf{r}, p}\right\rangle\left\langle e_{\mathbf{r}, p}\right| \\
& \times \widehat{U}_{0}^{\dagger}\left(t^{\prime \prime}\right) \widehat{E}_{p}^{(+) S P}\left(\mathbf{r}^{\prime \prime}\right)\left|e_{\mathbf{r}^{\prime \prime}, p}\right\rangle\langle g| \widehat{U}_{0}\left(t^{\prime \prime}\right)
\end{aligned}
$$

where $\Delta t$ is the time required for the measurement. The effect of the unitary time development operators can be evaluated by transforming the localized basis vectors, $\left|e_{\mathbf{r}, p}\right\rangle$, to the Bloch basis vectors that are delocalized within a pixel. The Wannier and Bloch bases are both complete so $\sum_{\mathbf{r}}\left|e_{\mathbf{r}, p}\right\rangle\left\langle e_{\mathbf{r}, p}\right|$ can be replaced with $\sum_{\mathbf{q}}\left|\psi_{\mathbf{q}, p}\right\rangle\left\langle\psi_{\mathbf{q}, p}\right|$ and (12) can be substituted in $\left|e_{\mathbf{r}^{\prime \prime}, p}\right\rangle$ and $\left\langle e_{\mathbf{r}^{\prime}, p}\right|$. Using assumptions equivalent to those in Kimble and Mandel's photodetection theory [16] in the impulsive detector sensitivity limit [17] and the orthogonality of the Bloch states, a factor $\left|\int_{0}^{\infty} d \epsilon_{q} \exp \left(-i \epsilon_{q} \tau\right)\right|=$ $2 \pi \hbar \delta(\tau)$ is obtained. With $\tau=t^{\prime \prime}-t^{\prime}$, Eq. (18) can then be written as

$\widehat{F}_{1, n}^{H P}\left(t_{0}\right)=s \int_{t_{0}}^{t_{0}+\Delta t} d t \sum_{p, \mathbf{r} \in D_{n}} \widehat{E}_{p}^{(-)}(\mathbf{r}, t) \widehat{E}_{p}^{(+)}(\mathbf{r}, t)|g\rangle\langle g|$

where $\widehat{E}_{p}^{(+)}(\mathbf{r}, t)$ is the IP positive frequency electric field operator, $s=2 \pi \rho_{\epsilon}|\mu|^{2} / \hbar$ is the one-atom sensitivity to the absolute square of the field per unit time, and $\rho_{\epsilon}$ is the number of states per unit energy interval in the semiconductor. A Glauber ideal photodetector has a flat frequency response, equivalent to assuming that $s$ is constant, and we have followed that convention here. Absorption of photons in different pixels is only correlated through the wave function, so the multi-photon counting operators will be time ordered integrals of factors of the form (19).

Inside the detector the $z$-component of the wave vector acquires an imaginary part, $i \alpha_{k}$, due to absorption, and the speed of light becomes $c / n$ where $n$ is the index of refraction. Frequency and the component of the wave vector tangential to the interface is unchanged by entry into the detector. If a mode with free space wave vector $\mathbf{k}$ approaches the detector at an angle $\theta$ to its normal it is refracted to $\theta_{d}$ inside the detector so that $\sin \theta_{d}=$ $\sin \theta / n$ and $k_{z} \rightarrow n k \cos \theta_{d}+i \alpha_{k}$. For normal incidence in the paraxial approximation $\cos \theta_{d}=1$. The IP positive frequency electric field operator inside the photodetector is then

$$
\begin{aligned}
\widehat{E}_{p}^{(+)}(\mathbf{r}, t) & =i \sum_{\mathbf{k}} E_{k} \widehat{a}_{\mathbf{k}, p} \\
& \times \exp \left(i k_{x} x+i k_{y} y+i n k z-i k c t-\alpha_{k} z\right) .
\end{aligned}
$$

\section{Photon counting}

The response of an ideal photon counting detector to photon flux is discussed by Bondurant [17]. The detector must be thick enough to absorb all incident photons, so we can integrate over all $z$ inside the detector. To integrate (19) over $z$ we can convert the sum over atom positions to an integral using $\sum_{\mathbf{r}} \rightarrow \rho_{a} \int d^{3} r$ where $\rho_{a}$ is 
the density of atoms to give

$$
\widehat{F}_{1, n}^{H P}(t)=\int_{t_{0}}^{t_{0}+\Delta t} d t \int_{A_{n}} d x d y \widehat{w}_{1}(x, y, t)|g\rangle\langle g|
$$

where $A_{n}$ is the area of the $n^{\text {th }}$ pixel and

$$
\widehat{w}_{1}(x, y, t)=s \rho_{a} \sum_{p} \int_{0}^{\infty} d z \widehat{E}_{p}^{(-)}(\mathbf{r}, t) \widehat{E}_{p}^{(+)}(\mathbf{r}, t)
$$

is the first-order coincidence rate operator in [12] and [17.

The essence of Bondurant's calculation can be understood by consideration of a single mode field with wave vector $\mathbf{k}$ and definite polarization. Using (20) and (11), $\widehat{E}^{(-)} \widehat{E}^{(+)}=\hbar k c \widehat{a}^{\dagger} \widehat{a} / 2 \epsilon_{0} V=\left(\hbar k c / 2 \epsilon_{0}\right) \widehat{n}$ where $\widehat{n}=a^{\dagger} a / V$ decays inside the dielectric. Since $d \widehat{w}_{1}(\mathbf{r}, t)=$ $\left(s \rho_{a} \hbar k c / 2 \epsilon_{0}\right) \widehat{n} d z$ is the absorption probability per unit area per unit time over the distance $d z, d \widehat{n}=-d \widehat{w}_{1} / c=$ $-\left(s \rho_{a} \hbar k / 2 \epsilon_{0}\right) \widehat{n} d z$ implies that

$$
\alpha_{k}=\frac{s \rho_{a} \hbar k}{4 \epsilon_{0}} .
$$

Using Equations (11) and (20), integration of (22) over a thick detector then gives $\widehat{w}_{1}=s \rho_{a} \int_{0}^{\infty} d z \widehat{E}^{\dagger} \widehat{E}=c \widehat{n}$, that is energy density is replaced with number density, implying that a thick photodetector counts photons.

More generally integration of (22) over $z$ with substitution of (20) and (11) gives [17]

$$
\begin{aligned}
\widehat{w}_{1}(x, y, t) & =\frac{s \rho_{a} \hbar c}{2 \epsilon_{0} V} \sum_{p, \mathbf{k}, \mathbf{k}^{\prime}} \frac{\sqrt{k k^{\prime}} \widehat{a}_{\mathbf{k}, p}^{\dagger} \widehat{a}_{\mathbf{k}^{\prime}, p}}{\alpha_{k}+\alpha_{k^{\prime}}+i n\left(k-k^{\prime}\right)} \\
& \times \exp \left[i\left(k_{x}-k_{x}^{\prime}\right) x+i\left(k_{y}-k_{y}^{\prime}\right) y\right. \\
& \left.-i\left(k-k^{\prime}\right) c t\right] .
\end{aligned}
$$

A photon should be detected with certainty if we sum over all pixels and count for a time $T$. To test this we can evaluate $\sum_{n} \widehat{F}_{1, n}^{H P}$ for $t=0$ and $\Delta t=T$. Substituting (24) in (19) and summing over pixels we get a factor $\delta_{k_{x}, k_{x}^{\prime}} \delta_{k_{y}, k_{y}^{\prime}} \delta_{k, k^{\prime}}$ inside the $\mathbf{k}$ and $\mathbf{k}^{\prime}$ sum with the result

$$
\sum_{n} \widehat{F}_{1, n}^{H P}=\sum_{p, \mathbf{k}} \widehat{a}_{\mathbf{k}, p}^{\dagger} \widehat{a}_{\mathbf{k}, p}|g\rangle\langle g| .
$$

Thus a photon is counted with certainty if the detector is big enough and it is operated over a long enough time.

The $k$-dependent factor in (24) can be expanded in powers of the relative differential photon frequency

$$
\delta_{k}=\left(k-k_{0}\right) / k_{0}
$$

where $k_{0} c$ is the center frequency of the pulse. To first order in $\delta_{k}$

$$
\begin{aligned}
\widehat{w}_{1}(x, y, t) & =\frac{c}{V} \sum_{p, \mathbf{k}, \mathbf{k}^{\prime}} \widehat{a}_{\mathbf{k}, p}^{\dagger} \widehat{a}_{\mathbf{k}^{\prime}, p} \\
& \times \exp \left[i\left(k_{x}-k_{x}^{\prime}\right) x+i\left(k_{y}-k_{y}^{\prime}\right) y\right. \\
& \left.-i\left(k-k^{\prime}\right) c(t-\tau)\right]
\end{aligned}
$$

where $\tau$ is a small delay of the order of a few optical cycles [17]. This can be written as

$$
\widehat{w}_{1}(x, y, t)=c \widehat{n}(x, y, 0, t-\tau)
$$

where $\widehat{n}(\mathbf{r}, t)$ given by (4) is the number density operator. This implies that a photon counting array measures photon flux density. The factor $\exp \left(-i k_{0} c t\right)$ disappears from (28), so $\widehat{n}$ describes the envelope function of the pulse and is unaffected by its center frequency. The $z$ integration is performed independently in each pixel and $t>t^{\prime}$ and $t<t^{\prime}$ are equally likely, so the two-photon correlation operator is

$\widehat{w}_{2}\left(x, y, t ; x^{\prime}, y^{\prime}, t^{\prime}\right)=\frac{1}{2 !} c^{2} \widehat{n}\left(x^{\prime}, y^{\prime}, 0, t^{\prime}-\tau\right) \widehat{n}(x, y, 0, t-\tau)$.

According to (11), the one-photon POVM is the partial trace of (21) over the atoms, $\widehat{P}_{1, n}=\operatorname{Tr}_{\mathcal{A}}\left(\widehat{\rho}_{\mathcal{A}} \widehat{F}_{1, n}^{H P}\right)$. For a photodetector initially in its ground state $\widehat{\rho}=\widehat{\rho}_{\mathcal{P}} \otimes$ $|g\rangle\langle g|$ so that the trace operation just eliminates $|g\rangle\langle g|$ giving

$$
\widehat{P}_{1, n}\left(t_{0}\right)=\int_{t_{0}}^{t_{0}+\Delta t} d t \int_{A_{n}} d x d y \widehat{w}_{1}(x, y, t) .
$$

If two photons are absorbed the POVM is

$$
\begin{aligned}
\widehat{P}_{2, n, n^{\prime}}\left(t_{0}\right) & =\int_{t_{0}}^{t_{0}+\Delta t} d t \int_{A_{n}} d x d y \\
& \times \int_{t_{0}}^{t} d t^{\prime} \int_{A_{n^{\prime}}} d x^{\prime} d y^{\prime} \widehat{w}_{2}\left(x, y, t ; x^{\prime}, y^{\prime}, t^{\prime}\right) .
\end{aligned}
$$

These are coarse-grained photon position operators. The pixel location can be identified with the $x$ and $y$ position of the photon, while the $z$ position of the photon is determined by the measurement time.

\section{Limitations of the model}

The photon counting array considered here will not count photons in all beams configurations. In the extreme case of propagation away from the detector, no photons will be counted at all. For modes not normal to the detector surface some photons will be counted in pixels adjacent to their entry pixel, and spatial resolution will be reduced. To go beyond the paraxial approximation or for application to beams not normal to the detector surface, (20) and equations derived from it would have to be generalized.

A real photodetector has a frequency dependent quantum efficiency less that one that should be corrected for when the photon number is determined from the number of electron-hole pairs created. If it is not possible to determine whether more than one photon was absorbed in a particular pixel, the photon density should be low enough that multiple photon absorption in a single pixel is unlikely. 
It was assumed in (6) that an absorbed photon has definite polarization. This was proved to be the case for normal incidence with $E_{z}$ neglected in [16]. In general, the Hermitian tensor $\operatorname{Tr}\left\{\widehat{\rho} \widehat{E}_{p}^{(-)}(\mathbf{r}, t) \widehat{E}_{p^{\prime}}^{(+)}(\mathbf{r}, t)\right\}$ can be diagonalized so "there always exist a set of three (complex) orthogonal polarization vectors such that the field components in these directions are statistically uncorrelated" [12]. If we use these polarization vectors as a basis, the diagonal form is exact.

To first order in an expansion over the relative differential photon frequency a thick photodetector counts photons with a small delay time of a few optical cycles. The delay $\tau$ has a simple physical interpretation. It is due to the $z$-integral that samples the square of the field over a distance of a few penetration depths, and these photons arrived at an earlier time.

Second order terms in the $\delta_{k}$ expansion that go as $\delta_{k}^{2}$ and $\delta_{k} \delta_{k^{\prime}}$ result in $\omega_{0}^{-2} \widehat{a}\left(\partial^{2} \widehat{a} / \partial t^{2}\right)$ and $\omega_{0}^{-2}(\partial \widehat{a} / \partial t)(\partial \widehat{a} / \partial t)$ in real space where $\omega_{0}=c k_{0}$. There is also a term that goes as $\delta_{k}\left(k_{x}^{2}+k_{y}^{2}\right) / k_{0}^{2}$ that gives $\omega_{0}^{-1} k_{0}^{-2}(\partial \widehat{a} / \partial t)\left(\partial^{2} \widehat{a} / \partial x^{2}+\partial^{2} \widehat{a} / \partial y^{2}\right)$ in real space. These higher order terms depend on the rate of change of the pulse envelope relative to the optical frequency and are negligible in most experiments.

For a single atom the factor $\sqrt{k}$ gives a nonlocal relationship between field and number amplitude such that the field at $\mathbf{r}^{\prime}$ of a photon at $\mathbf{r}$ is proportional to $\left|\mathbf{r}-\mathbf{r}^{\prime}\right|^{-7 / 2}[\underline{3}, 18]$. This nonlocality is almost completely eliminated by integration over $z$, and is neglected here.

\section{E. Photon state and wave function}

As an illustration (28) and (29) will be applied to paraxial one and two photon states at normal incidence. For a one-photon pulse, if the time required for a measurement is less than the pulse length, detection of either 1 or 0 photons is possible. The probability to count one photon between times $t_{0}$ and $t_{0}+\Delta t$ is $P_{1}=\sum_{n} P_{1, n}$ given by (30) while the zero-photon probability is $1-P_{1}$. If this intermediate photon number is read out, the wave function collapses to a 0 or 1 photon state [19]. To avoid the complications of intermediate collapse we will only consider measurement times greater than the pulse length.

A HP one-photon pure state is of the form

$$
\left|\psi_{1}\right\rangle=\sum_{\mathbf{k}, \sigma} c_{\mathbf{k}, \sigma}|\mathbf{k}, \sigma\rangle
$$

where $\sum_{\mathbf{k}, \sigma}\left|c_{\mathbf{k}, \sigma}\right|^{2}=1$. The expectation value of the photon flux density operator is

$$
\begin{aligned}
& \left\langle\psi_{1}\left|\widehat{w}_{1}(x, y, t)\right| \psi_{1}\right\rangle \\
& =c \sum_{\sigma}\left\langle\psi_{1}\left|\widehat{a}_{\sigma}^{\dagger}(x, y, t)\right| 0\right\rangle\left\langle 0\left|\widehat{a}_{\sigma}(x, y, t)\right| \psi_{1}\right\rangle .
\end{aligned}
$$

The photon wave function will be defined here as the projection of $\left|\psi_{1}\right\rangle$ onto the one-photon state (3) with polarization $\sigma$ localized at $\mathbf{r}$ at the measurement time $t$, that is

$$
\begin{aligned}
\psi_{\sigma}(\mathbf{r}, t) & =\left\langle\mathbf{r}, t, \sigma \mid \psi_{1}\right\rangle \\
& =\sum_{\mathbf{k}} c_{\mathbf{k}, \sigma} \frac{\exp (-i \mathbf{k} \cdot \mathbf{r}+i k c t)}{\sqrt{V}} .
\end{aligned}
$$

At each point in space $\psi_{\sigma}$ has two components corresponding to the two transverse photon polarizations. For a CP paraxial beam the unit vectors at $\theta=0$ reduce to $\mathbf{e}_{ \pm 1}^{(0)}=(\widehat{\mathbf{x}}+i \sigma \widehat{\mathbf{y}}) / \sqrt{2}$ and, together with $\widehat{\mathbf{z}}$, can be identified with the $\mathbf{e}_{p}$ basis. For LP we can take $\chi=-\phi$ so that $\mathbf{e}_{1}^{(-\phi)}=\widehat{\mathbf{x}}$ and $\mathbf{e}_{-1}^{(-\phi)}=\widehat{\mathbf{y}}$ at $\theta=0$. In either case

$$
\left\langle\psi_{1}\left|\widehat{w}_{1}(x, y, t)\right| \psi_{1}\right\rangle=c \sum_{\sigma}\left|\psi_{\sigma}(x, y, 0, t)\right|^{2},
$$

is photon flux density.

A two-photon HP state vector is of the form

$$
\left|\psi_{2}\right\rangle=\sum_{\mathbf{k}, \mathbf{k}^{\prime}, \sigma, \sigma^{\prime}} c_{\mathbf{k}, \sigma ; \mathbf{k}^{\prime}, \sigma^{\prime}}\left|\mathbf{k}, \sigma ; \mathbf{k}^{\prime}, \sigma^{\prime}\right\rangle
$$

If we define the symmetric two-photon wave function

$$
\begin{aligned}
\psi_{\sigma, \sigma^{\prime}}\left(\mathbf{r}, t ; \mathbf{r}^{\prime}, t^{\prime}\right) & =\frac{1}{\sqrt{2 !}}\left\langle\emptyset\left|\widehat{a}_{\sigma}(\mathbf{r}, t) \widehat{a}_{\sigma^{\prime}}\left(\mathbf{r}^{\prime}, t^{\prime}\right)\right| \psi_{2}\right\rangle \\
& =\frac{1}{\sqrt{2 !} V} \sum_{\mathbf{k}, \mathbf{k}^{\prime}}\left(c_{\mathbf{k}, \sigma ; \mathbf{k}^{\prime}, \sigma^{\prime}}+c_{\mathbf{k}^{\prime}, \sigma^{\prime} ; \mathbf{k}, \sigma}\right) \\
& \times \exp \left[i\left(-\mathbf{k} \cdot \mathbf{r}-\mathbf{k}^{\prime} \cdot \mathbf{r}^{\prime}+k c t+k^{\prime} c t^{\prime}\right)\right],
\end{aligned}
$$

the two-photon coincidence rate is

$$
\begin{aligned}
& \left\langle\psi_{2}\left|\widehat{w}_{2}\left(x, y, t ; x^{\prime}, y^{\prime}, t^{\prime}\right)\right| \psi_{2}\right\rangle \\
& =c^{2} \sum_{\sigma, \sigma^{\prime}}\left|\psi_{\sigma, \sigma^{\prime}}\left(x, y, 0, t ; x^{\prime}, y^{\prime}, 0, t^{\prime}\right)\right|^{2} .
\end{aligned}
$$

For example, if the photons are produced by spontaneous parametric down conversion (SPDC) it was found that their positions are highly correlated [8]. For type II SPDC the polarization of the photons is orthogonal, while for type I the polarizations are the same. Both these effects can be described by (38). In the photon counting experiment considered here polarization was not measured, and should be summed over. Knowledge of photon polarization comes from knowledge of the beam properties rather than from the photodetection process.

\section{F. Collapse}

We will start by considering a pure state, $\left|\psi_{1}\right\rangle|g\rangle$, that initially contains one-photon and assume that an e-h pair has been detected in pixel $n$. The extended system of photons plus detector atoms satisfies the usual rules of 
quantum mechanics. In the IP this state vector evolves with time to $|\Psi(t)\rangle=\widehat{U}^{(1)}(t)\left|\psi_{1}\right\rangle|g\rangle$ and collapses to the final state $\left|\Psi_{1, f}^{I P}\right\rangle=\widehat{F}_{1, n}^{I P}|\Psi(t)\rangle$ after a measurement. Since its final and initial states are the same, $\widehat{F}_{1, n}^{I P}=\widehat{F}_{1, n}^{S P}$ is given by (6) so that

$$
\left|\Psi_{1, f}^{I P}\right\rangle \propto \sum_{\sigma= \pm 1, \mathbf{r} \in D_{n}}\left|e_{\mathbf{r}, \sigma}\right\rangle\left\langle e_{\mathbf{r}, \sigma}\left|\widehat{U}^{(1)}(t)\right| \psi_{1}\right\rangle|g\rangle .
$$

If $\psi_{\sigma}(x, y, 0, t)$ is uniform over the area of a pixel and polarization isn't measured the normalized collapsed state vector simplifies to

$$
\left|\Psi_{1, f}^{I P}\right\rangle=\frac{1}{\sqrt{2 N}} \sum_{\sigma, \mathbf{r} \in D_{n}}\left|e_{\mathbf{r}, \sigma}\right\rangle|\emptyset\rangle
$$

where $N$ is the number of atoms in the $n^{\text {th }}$ pixel. If $J$ photons are detected and the wave function is approximately uniform over a pixel the system collapses to the state

$$
\left|\Psi_{J, f}^{I P}\right\rangle=\prod_{i=1}^{J}\left(\frac{1}{\sqrt{2 N}} \sum_{\sigma_{i}, \mathbf{r}_{i} \in D_{n_{i}}}\left|e_{\mathbf{r}_{i}, \sigma_{i}}\right\rangle\right)|\emptyset\rangle .
$$

\section{DISCUSSION}

The one-photon flux density operator (28) for helicity $\sigma$ can be written as $c|\mathbf{r}, t, \sigma\rangle\langle\mathbf{r}, t, \sigma|$ evaluated at $z=0$. The Hermitian position operator $\widehat{\mathbf{r}}_{\sigma}=$ $\int d^{3} r \mathbf{r}|\mathbf{r}, t, \sigma\rangle\langle\mathbf{r}, t, \sigma|$ with eigenvectors $|\mathbf{r}, t, \sigma\rangle$ and eigenvalues $\mathbf{r}$ and $\sigma$ can be defined such that the usual rules of quantum mechanics are formally satisfied. For two photons the projection operators can be written as $\left|\mathbf{r}, t, \sigma ; \mathbf{r}^{\prime}, t^{\prime}, \sigma^{\prime}\right\rangle\left\langle\mathbf{r}, t, \sigma ; \mathbf{r}^{\prime}, t^{\prime}, \sigma^{\prime}\right|$, consistent with the $1 \mathrm{D}$ multi-photon POVMs defined by Tsang [9]. There are in principle many ways to construct a POVM, since any partition of the identity is allowed. However, an exactly localized position eigenvector is an equally weighted sum over all wave vectors, and this form arose naturally here. But there is an important deviation from the usual rules; collapse is not to this position eigenvector.

By the usual rules of quantum mechanics, if position of a photon with polarization $\sigma$ is measured at time $t$ the system should collapse to the instantaneously localized state, $|\mathbf{r}, t, \sigma\rangle$. Since all wave vectors are equally likely in a localized state such as (3), both incoming and outgoing states are required [7]. In a photon counting experiment there is no outgoing wave and collapse to an exactly localized state is not possible. In place of the usual rules, here we have used the theory of generalized observables that separates the calculation of probabilities from collapse. With this formalism we found that a photon counting array detector measures coarse grained photon position, while collapse is to the zero photon vacuum state. Some theorists would insist we say "absorption position" was measured, but the term "photon position" is widely used.

Definite helicity basis states were selected because all wave vectors can be included and helicity is Lorentz invariant. States with a definite polarization direction in real space exclude longitudinal wave vectors and thus can not be localized. The definite helicity unit vectors have definite total angular momentum parallel to some $z$-axis [1]. Selecting the $z$-axis is analogous to selecting the quantization axis for electron spin that is arbitrary in the absence of symmetry breaking, but can be fixed by a magnetic field. For the photon, symmetry can be broken by the beam axis.

In nonrelativistic quantum mechanics the real space wave function is both $\langle\mathbf{r} \mid \psi\rangle$ and a solution to the particle's wave equation. For photons these are not the same thing, the former being a scalar while the latter is a vector whose $\mathbf{k}^{\text {th }}$ term is weighted as $\sqrt{k}$. Here the photon wave function is defined in the former sense, that is as the projection of the photon state vector onto a basis of the localized states. The photon wave function is $\psi_{\sigma}(\mathbf{r}, t)=\left\langle\mathbf{r}, t, \sigma \mid \psi_{1}\right\rangle$ and the photon flux density measured in a photon counting experiment is $c \sum_{\sigma}\left|\psi_{\sigma}(x, y, 0, t)\right|^{2}$. The quantum electrodynamic state vector is used to describe the photon state and a wave function that satisfies Maxwell's equations is not required. This choice of wave function is consistent with Chan et al [6], with Tsang [9], and with the discussion of position eigenvectors in [7].

In summary, a photon position POVM for a photon counting experiment has been derived by performing a partial trace over the ancillary Hilbert space of the photodetector atoms. It was found that the photon counting probability equals the photon flux density integrated over the area of the pixel and the time taken for a measurement. This is, of course, as expected, since a photon counting array is designed to measure photon position. If the photon wave function is defined as the projection of its state vector onto the localized states, photon flux density is its absolute square multiplied by the speed of light. The photons are destroyed in this measurement, but the distribution of positions and times at which they are detected is a measure of their probability density in the incoming pulse.

Acknowledgements: The author thanks the Natural Sciences and Engineering Research Council for financial support. 
[3] I. Bialynicki-Birula, in Progress in Optics XXXVI, edited by E. Wolf (Elsevier, 1996).

[4] I. Bialynicki-Birula, Phys. Rev. Lett. 80, 5247 (1998).

[5] M. O. Scully and M. S. Zubairy, Quantum Optics (Cambridge Univ. Press, 1997).

[6] K.W. Chan, C.K. Law and J.H. Eberly, Phys. Rev. Lett. 88, 100402 (2002).

[7] M. Hawton, Phys. Rev. A 78, 012111 (2008).

[8] M. N. O'Sullivan-Hale, I. A. Khan, R. W. Boyd, and J. C. Howell, Phys. Rev. Lett. 94, 220501 (2005).

[9] M. Tsang, Phys. Rev. Lett. 102, 253601 (2009).

[10] K. Kraus in The Uncertainty Principle and Foundations of Quantum Mechanics, edited by W. C. Price and S. S. Chissick (Wiley, 1977), pp. 293-320.

[11] M. Hawton and W. E. Baylis, Phys. Rev. A, 71, 033816
(2005).

[12] R. J. Glauber, Phys. Rev. 130, 2529 (1963).

[13] H. Haug and S. W. Koch, Quantum Theory of the Optical and Electronic Properties of Semiconductors (World Scientific, 1990), Ch. 3

[14] Ref. [5], Ch6

[15] J. J. Sakurai, Modern Quantum Mechanics (AddisonWesley, 1985), p. 326.

[16] H. J. Kimble and L. Mandel, Phys. Rev. A 30, 844 (1984).

[17] R. S. Bondurant, Phys. Rev. A 32, 2797 (1985).

[18] W. O. Amrein, Helv. Phys. Acta 42, 149 (1969).

[19] M. Ueda, N. Imoto, and T. Ogawa, Phys. Rev. A 41, 3891 (1990). 